Results 125/208 registered women consented to participate $(60.1 \%), 64.4 \%$ of the recruitment target (125/194). Recruitment was an iterative process and the target number (48) was reached in wave 4. Dropout rates were high, 67/125 (53.6\%) at $12 \mathrm{w}$ and $59 / 125(47.2 \%)$ at $6 \mathrm{~m}$. While the study was not powered to test effectiveness, $26.6 \%$ of women randomised to the intervention group were non-smokers at 12 weeks versus $16.6 \%$ of controls. Higher quit rates were observed in the intervention group, $(41.7 \%$ intervention, $25.8 \%$ control) in those who attended at least one delivery session (89). Differences in quit rates were not sustained at 6/12. High acceptability of trial processes and intervention delivery were reported.

Conclusion Recruitment to a community based smoking cessation pilot RCT for disadvantaged women was challenging but feasible. Challenges to recruitment and lessons learned will be discussed. A positive direction of effect in favour of the intervention was noted. Measures to improve retention rates are needed in a future definitive intervention trial.

\section{OP14 THE IMPACT OF HEALTH SYSTEM SUPPORT AND MIGRATION RELATED RISK FACTORS ON DUAL AND POLY-TOBACCO USE AMONG MALE ADULTS IN 15 LOW AND MIDDLE-INCOME COUNTRIES - IMPLICATIONS FOR GLOBAL TOBACCO CONTROL}

THC Chen* ${ }^{*}$ CM Millett, FTP Filippidis. School of Public Health, Imperial College London, London, UK

\subsection{6/jech-2020-SSMabstracts. 14}

Background Concurrently using two (dual users) or more than two (poly tobacco users) tobacco products is common in lowand middle-income countries, especially among men, and has been linked to increased health risks and nicotine addiction compared to single tobacco product use. While substantial inequalities persists with high tobacco use prevalence among disadvantaged groups, very little is known about the impact of health care support and migration status on tobacco use patterns among these population. This study aims to explore socioeconomic, health care and migration related risk factors of ploy tobacco users among men in 15 low-and middleincome countries.

Methods Data from 15 countries around the world were obtained from the most recent wave of the Demographic and Health Survey (DHS), collected between 2015 and 2018. A total of 155,425 men aged 15-49 were surveyed. Outcomes included single, dual and poly use, examined by education, wealth, health system support and migration status. Migration defined as whether the respondent slept or reside away from home in the last 12 months. Multilevel models were used to estimate the effect of covariates on tobacco use with along with fixed and random parameters.

Results In all studied countries, prevalence of dual and polytobacco use was highest in respondents with low socioeconomic status (SES). SES gradients in dual and poly use by education $(\mathrm{RR}=0.64$; 95\% CI: $0.55-0.67$ for higher education vs illiterates) and wealth index $(\mathrm{RR}=0.15 ; 95 \% \mathrm{CI}$ : 0.11-0.20 for richest vs poorest) were observed. Furthermore, results showed higher risks of being a poly user among migrant men $(\mathrm{RR}=1.70 ; 95 \% \mathrm{CI}: 1.65-1.71)$ and $\mathrm{a}$ lower risk against dual use for those covered by health insurance $(\mathrm{RR}=0.82 ; 95 \% \mathrm{CI}$ : 0.79-0.85). However, dual and poly tobacco use were not associated with a country's purchasing power parity.

Conclusion Positive associations were found between dual and poly tobacco use among male respondents with lower SES and migration backgrounds, whereas respondents protected by health care system were associated negatively. This is the first study to examine the impact of health system support and migration status among men in low and middle-income countries. Findings imply that policy makers should consider targeting populations with migration background and those with reduced access to health care to tackle with smoking inequality in tobacco control science.

\section{OP15 EXPLORING THE IMPACT OF SMOKE-FREE LEGISLATION ON EXPOSURE TO SECOND-HAND SMOKE AMONG NON-SMOKING ADULTS IN ENGLAND}

C Mathew*, C Kypridemos. Department of Public Health and Policy, University of Liverpool, Liverpool, UK

\subsection{6/jech-2020-SSMabstracts. 15}

Background In the presence of mounting evidence on the adverse effects of second-hand smoke (SHS) to non-smokers, England implemented smoke-free legislation on 1 July 2007 that rendered all public enclosed spaces smoke-free. As England considers becoming smoke-free by 2030, an evaluation of the existing smoke-free policy in England utilizing recent data becomes paramount in informing policymakers who are debating an expansion of the legislation in England. This project sought to examine trends in exposure to SHS among adult non-smokers in England from 2003-2015 in order to determine whether the legislation produced changes in SHS exposure among non-smoking adults and whether the policy had a differential impact by sex and socioeconomic status (SES).

Methods This study was an interrupted time series analysis of data on self-reported exposure to SHS among adult non-smokers that were obtained from Health Survey England and spanned the period between 2003 and 2015. The study used regression methods to examine trends in not only exposure to SHS as a binary variable but also the number of hours of exposure to SHS both before and after the ban. The analysis was conducted for the general adult non-smoking population as well as by sex and SES, using the quintile groups of the index of multiple deprivation.

Results The odd of exposure to SHS was falling annually by 9.1\% (95\% CI: $6.7 \%$ to $11.5 \%$ ), in relative terms, before the implementation of the smoke-free policy. The odds dramatically reduced by $189.4 \%$ (95\% CI: $100.2 \%$ to $318.7 \%$ ) as a result of the policy, but slowly increased since then by $1.7 \%(95 \%$ CI: -3.0 to $6.2 \%$ ) annually. The modelled prevalence of SHS exposure, declined drastically by approximately 50\%, from $40.5 \%$ (95\% CI: $34.5 \%-46.9 \%$ ) to $19.1 \%$ (95\% CI: $11.2 \%$ $30.6 \%)$, in the immediate aftermath of the ban. Nevertheless, the number of hours of SHS exposure declined after the ban. The policy was most effective in men, and it reduces both absolute and relative sex inequalities. When socioeconomic inequalities were considered, the policy was more effective in the most deprived group, and reduced absolute socioeconomic inequality, however, absolute inequality was increased as a result of the policy. The analysis was performed in SPSS v24.

Conclusion While the population-wide decline in SHS exposure following the ban is a treatment to the success of smoke- 
free legislation, the persisting relative inequalities in SHS exposure by SES highlight the need for continued investment in tobacco control policies.

\section{OP16 DEVELOPING A SMOKE-FREE HOME INTERVENTION FOR NEONATAL INTENSIVE CARE UNITS - A QUALITATIVE STUDY}

${ }^{1} \mathrm{CJ}$ Notley*, ${ }^{1} \mathrm{TJ}$ Brown, ${ }^{2} \mathrm{~A}$ Nichols, ${ }^{3} \mathrm{~L}$ Bauld, ${ }^{4} \mathrm{~W}$ Hardeman, ${ }^{5} \mathrm{E}$ Boyle, ${ }^{5} \mathrm{M}$ Hubbard ${ }^{4} \mathrm{~F}$ Naughton, ${ }^{6} \mathrm{M}$ Ussher, ${ }^{1,2} \mathrm{P}$ Clarke, ${ }^{7} \mathrm{R}$ Holland, ${ }^{8} \mathrm{~S}$ Orton. ${ }^{1}$ Norwich Medical School, University of East Anglia, Norwich, UK; ${ }^{2}$ Norfolk and Norwich University Hospitals NHS Foundation Trust, Norwich, UK; ${ }^{3}$ Edinburgh University, Edinburgh, UK; ${ }^{4}$ School Of Health Sciences, University of East Anglia, Norwich, UK; ${ }^{5}$ University Hospitals of Leicester NHS Trust, Leicester, UK; ${ }^{6}$ St Georges, University of London and University of Stirling, London and Stirling, UK; ${ }^{7}$ Leicester Medical School, Leicester, UK; ${ }^{8}$ University of Nottingham, Nottingham, UK

\subsection{6/jech-2020-SSMabstracts. 16}

Background Babies born to smokers weigh on average $200 \mathrm{~g}$ less than those born to non-smokers and are at $40 \%$ higher risk of being born preterm. The relative risk of admission to Neonatal Intensive Care units (NICU) for infants of smokers is increased by at least $20 \%$. Parents of infants admitted to NICU may feel helpless and overwhelmed at a time when their baby is critically ill. Stopping smoking, or remaining abstinent, is one of the few things that parents can do to significantly improve the longer-term recovery and health of their offspring, yet stressed parents are at increased risk of smoking relapse. NICU admission may represent a 'teachable moment' where parents are receptive to smoking cessation.

Methods Qualitative focus groups and interviews with parents and family members of babies admitted to NICUs. Participants were purposively sampled $(n=60)$ from NICUs across two large UK teaching hospitals, seeking maximum variation in smoking status, parental/familial status, ethnicity and socioeconomic status. Qualitative topic guides sought feedback on potential intervention approaches, considering 'who' might introduce, 'what' might be the content, and 'when' an intervention might be delivered. Data were collected face to face by dedicated neonatal research nurses. All data were audio recorded and transcribed verbatim. Inductive thematic analysis of data was conducted by two members of the research team, independently reviewing coding to reach consensus on emergent themes.

Results Parents appear amenable to smoking cessation and express surprise that the subject is not addressed. Immediate addressing of smoking status would not be appropriate on acute admission to NICU due to stress and concerns regarding the newborn, but timely support is needed to reach those willing to quit, and those who had quit during pregnancy but were at high risk of relapse. Support might best be delivered by a NICU nurse with specialist training. Support with cessation and relapse prevention through information about smokefree homes, nicotine replacement therapy and/or support to use nicotine in significantly less harmful ways (e.g. vaping) were identified as promising routes for intervention. Parents welcomed ongoing support following discharge from NICU and were amenable to digital options.

Conclusion There is presently little dedicated support for smoking cessation, relapse prevention or smoke-free homes for families of NICU babies. Parents are amenable to support and consider a focus on smoke-free homes as a less stigmatising way in which smoking may be discussed and cessation promoted to improve the health of premature babies.

\section{OP17 EXAMINING INEQUALITY IN TRIALS OF SMOKING CESSATION INTERVENTIONS DELIVERED IN PRIMARY CARE: CRITIQUE AND REANALYSIS OF COCHRANE REVIEWS}

\author{
1,2JM Birch*, ${ }^{2} \mathrm{H}$ Dambha-Miller, ${ }^{1,2}{ }^{2} \mathrm{SJ}$ Griffin, ${ }^{2} \mathrm{~GB}$ Hutton, ${ }^{2} \mathrm{MP}$ Kelly, ${ }^{2} \mathrm{AL}$ Kinmonth. ${ }^{1} \mathrm{MRC}$ \\ Epidemiology Unit, University of Cambridge, Cambridge, UK; ${ }^{2}$ Department of Public Health \\ and Primary Care, University of Cambridge, Cambridge, UK

\subsection{6/jech-2020-SSMabstracts. 17}

Background Tobacco smoking is a major cause of chronic disease and premature mortality. Its effects are socially patterned. Observational studies show that low socioeconomic status [SES] is associated with higher smoking prevalence and lower cessation rates. Interventions in primary care may improve or exacerbate health inequalities depending on socioeconomic patterning of access and uptake. Data on the impact of trials of smoking cessation interventions delivered in primary care on health inequalities by SES have not been synthesised. We examined the impact of smoking cessation interventions delivered in primary care on inequalities in health by socioeconomic status.

Methods We searched the Cochrane database of systematic reviews from inception until June 2019. We included reviews of trials of smoking cessation interventions delivered in primary care and published in English.

Results We identified eight Cochrane reviews (413 studies). Eighty five studies included an intervention delivered in primary care. Interventions were: behavioural, (very) brief advice, and pharmacological (including nicotine replacement therapy). Full texts were accessed for 70 studies; 17 reported an SES measure. Two studies targeted low-SES groups. There was heterogeneity in SES measures used across the studies, which included household income, occupational level and social class. Three studies analysed SES as a predictor of effectiveness of the smoking cessation intervention; none found that effectiveness differed by SES.

Discussion This summary and critique of Cochrane reviews demonstrates that trials of smoking cessation interventions delivered in primary care are not designed to allow analysis of effects by measures of SES. Studies rarely reported SES of participants at baseline and hardly ever as a predictor of smoking cessation. Our work highlights the need for routine reporting of SES amongst trials and greater consensus in included measures. Consistent reporting of a core set of SES indicators will enable testing of similarities between trial groups and differential effects by SES.

\section{OP18 FROM SMOKING-PERMITTED TO SMOKEFREE PRISONS: A 3-YEAR EVALUATION OF THE CHANGES IN OCCUPATIONAL EXPOSURE TO SECOND-HAND SMOKE ACROSS A NATIONAL PRISON SYSTEM}

${ }^{1} S$ Semple*, ${ }^{2} E$ Demou, ${ }^{1} \mathrm{R}$ Dobson, ${ }^{2} \mathrm{H}$ Sweeting, ${ }^{3} \mathrm{~S}$ Sidwell, ${ }^{1} \mathrm{~A}$ Brown, ${ }^{1} \mathrm{R}$ O'Donnell, ${ }^{1} \mathrm{~K}$ Hunt. ${ }^{1}$ Institute for Social Marketing and Health, University of Stirling, Stirling, UK; ${ }^{2} \mathrm{MRC}$ CSO SPHSU, University of Glasgow, Glasgow, UK; ${ }^{3}$ Scottish Prison Service, SPS, Edinburgh, UK

\subsection{6/jech-2020-SSMabstracts. 18}

Background Prisons were one of the only workplaces where smoking continued to be permitted after the smoking ban in indoor public places in Scotland in 2006. Hence, the prison workforce remained potentially exposed to secondhand smoke 